\title{
Factores que intervienen en el nivel de ausentismo del personal que labora en un centro de llamado de Barranquilla (Colombia)
}

\author{
Margel Parra1, Nelson Lay ${ }^{2}$, Karen Payares ${ }^{3}$ y Arleth Pareja ${ }^{3}$ \\ (1) Universidad de la Costa, Facultad de Ciencias Humanas y Sociales, Barranquilla - Colombia. \\ (correo-e: mparra3@cuc.edu.co) \\ (2) Universidad Andrés Bello, Facultad de Educación y Ciencias Sociales, Santiago - Chile. \\ (correo-e: nelson.lay@unab.cl) \\ (3) Universidad de la Costa, Facultad de Ciencias Económicas, Barranquilla - Colombia. \\ (correo-e: kpayares4@cuc.edu.co; arlet.paredes@gmail.com;)
}

Recibido Jun. 17, 2020; Aceptado Ago. 17, 2020; Versión final Oct. 7, 2020, Publicado Dic. 2020

\begin{abstract}
Resumen
El objetivo de la presente investigación es analizar los factores que intervienen en el nivel de ausentismo del personal que labora en un centro de llamado de Barranquilla (Colombia). Se presenta una metodología cuantitativa de tipo correlacional y la aplicación de un cuestionario sobre las diversas dimensiones del ausentismo. La muestra consiste de 65 sujetos que laboran en un centro de llamado de la ciudad de Barranquilla. Los resultados muestran que existe un nivel de asociación significativo entre el ausentismo y las variables sociodemográficas de edad, cargo y tiempo dentro de la organización. Se concluye que el ausentismo presente en el centro de llamada objeto de estudio arrojó resultados elevados, lo cual se puede justificar al momento de analizar los niveles de riesgo, cultura organizacional y de compromiso, los cuales mostraron ser a su vez bastante negativos.
\end{abstract}

Palabras clave: ausentismo; centros de llamado; compromiso; cultura organizacional

\section{Factors involved in staff's absence from work at a call center in Barranquilla (Colombia)}

\begin{abstract}
The objective of the present research study is to analyze the factors involved in absence from work by employees, working at a call center in Barranquilla (Colombia). A quantitative correlational methodology and the application of a questionnaire on the various dimensions of absenteeism are applied. The sample consists of 65 subjects who work at the call center in Barranquilla. The results show that there is a significant correlation between absence from work and the socio-demographic variables of age, job position, and length of time working at the call center. It is concluded that work absence levels are high at the call center examined, based on analyzes of risk levels, organizational culture, and engagement, which showed to be highly negative.
\end{abstract}

Keywords: absenteeism; call centers; engagement; organizational culture 


\section{INTRODUCCIÓN}

Hoy en día, según Pinho (2019) las empresas enfocan sus esfuerzos para cumplir con los estándares de calidad del servicio prestado a los clientes, pues estas buscan posicionamiento, liderazgo, pero además principalmente la satisfacción de sus consumidores. Esta calidad de servicio es entonces un factor determinante para toda empresa prestataria de un servicio, tomando mucha relevancia al respecto, donde el desempeño laboral figura como el elemento principal para lograr dicha acción, tal como expresan Meesala y Paul (2018). Dichas organizaciones han reconocido que sólo ofreciendo un servicio de excelente calidad es como conquistarán esa satisfacción. En este ámbito buscan obtener ese posicionamiento, el cual basado en García et al (2017) podría considerarse como base fundamental para el crecimiento y fortalecimiento en el mercado donde se desenvuelve.

Bajo este contexto, comentan Elmorshidy et al (2015) y Agnihotri et al (2016) que la calidad de servicio en las organizaciones radica en orientar a los clientes en lograr satisfacer una necesidad, y además facilitarle el cumplimiento de sus expectativas individuales. Por otra parte, la atención brindada debe ser buena y personalizada. Por lo antes expuesto, es necesario optimizar los recursos en las empresas, con el fin de alcanzar las metas y objetivos propuestos. En este sentido, Comentan García et al. (2018) que a medida que las organizaciones confrontan las nuevas competencias exigidas por los mercados a nivel mundial, la apertura y el progreso de tecnología requieren con mayor disposición la permanencia de un capital humano apto y disponible en lugares y momentos específicos. De esta manera, explican Hernández-Sánchez et al. (2018) y Payares et al. (2020) que el talento humano posee una función clave para el cumplimiento de los objetivos planeados por la gerencia, ellos requieren de una planificación equilibrada que considere todos los recursos disponibles y necesarios para la empresa. De este modo, Cheshin, Amit \& Van Kleef (2018) relacionan de manera importante las acciones y actitudes del personal con la satisfacción del consumidor y la calidad de servicio y, por ende, el cumplimiento de las metas de los trabajadores.

Algunos autores como Zablah et al. (2016) o Deery et al. (2010) comentan que ciertamente existe una relación recíproca entre el recurso humano y la satisfacción de los consumidores; donde dicha relación se da desde adentro hacia afuera de manera más significante; implicando que son los trabajadores los que tienen mayor incidencia sobre la satisfacción de cliente y no viceversa. Esto permite recalcar la gran relevancia que tiene el personal para el logro de los objetivos estratégicos de cualquier organización. Habiendo comprendido la importancia que tienen los recursos humanos para las organizaciones, se considera, según aportan Payares et al. (2020), que es fundamental que estas tomen las medidas necesarias para combatir los diferentes problemas que afecten a los colaboradores en la realización de su labor y, por ende, puedan incurrir en una disminución de la calidad de los servicios prestados a la clientela. Se menciona el caso de Rompu, Wahyu y Abadi, (2018), quienes al estudiaron como diversas variables como la calidad de vida laboral, el clima organizacional o el estrés en el trabajo pueden tener un importante efecto sobre las dinámicas que se dan dentro de las organizaciones.

Entre estas problemáticas, se puede considerar que una de las más representativas es el ausentismo laboral, pues representa la constante ausencia de personal necesario para llevar a cabo las actividades de la empresa, tal como aporta Frick, Simmons y Stein (2018). Si bien es cierto que se debe reconocer que este fenómeno tiene múltiples causas pues, pueden ir desde una situación imprevista, como una eventual enfermedad, hasta la necesidad de atender circunstancias personales. No obstante, Rodríguez et al. (2019) y Souza et al. (2020) consideran que por más justificada que este esta ausencia, se genera una crisis y deterioro de los procesos para la empresa, situaciones que deben ser atendidas mediante un mayor esfuerzo de los trabajadores que sí se encuentran en su área de trabajo asumiendo roles o actividades que no son parte de sus funciones o para las que no están preparados.

En este mismo orden de ideas, Deery et al (2017) consideran que cuando esto se hace constante y repetitivo, se genera un problema a mayor escala que no solo produce una disminución significativa de la calidad de servicio brindada por la empresa, si no que crea un clima organizacional de mucha tensión. Lo mencionado permite según Stabio (2014) identificar la importancia de comprender por qué sucede este hecho en las organizaciones, donde se considera que tiene relación con diversidad de elementos tanto internos y externos que dan cabida a esta problemática. Muchos estudios han realizado un análisis de cómo afecta el ausentismo laboral a la calidad de los procesos que se llevan dentro de las instituciones del sector salud. Estas se caracterizan por los elevados niveles de estrés a los cuales los trabajadores se deben enfrentar constantemente; tal como se aprecia en investigaciones como las de Feldhaus et al. (2019) y Luengo et al. (2020).

En el caso de los llamados centros de llamado, Pillay, Buitendach y Kanengoni (2014) destacan que es el sector de más rápido crecimiento en el siglo XXI; siendo a su vez uno de los sectores con mayores índices de ausentismo e insatisfacción en la actualidad, tal como aporta Marshall (2019). Al citar a Arregui (2018) se puede mencionar que dentro de la región latinoamericana hay escasos estudios que generen aportes a dicha 
situación desde la realidad que se enfrenta en este lado del mundo, donde los hallazgos que se logran rastrear en redes especializadas permiten observar cómo los elementos culturales y legales hacen que varíen las causas de dicho objeto de estudio.

El caso de los centros de llamado de Barranquilla es sumamente significativo, pues según lo aportado por Duarte (2020) los mismos representan uno de sectores más importantes para la empleabilidad en la ciudad con un $8 \%$ de plazas de empleos de dicho sector en los primeros trimestres del año 2020. A su vez, el mismo estudio menciona que las tasas de ausentismo y las problemáticas que estos generan son sumamente elevadas presentando en su estudio un aumento del 3.5\% desde 2019 hasta 2020 dentro del centro de llamadas objeto de estudio de dicho investigador.

Ciertamente, Arregui (2018) comenta que es fundamental que para este tipo de organizaciones donde se manejan elevados niveles de exigencia y estrés constante, en aras de lograr un proceso de optimización y mejora continua es fundamental que se identifiquen todas las problemáticas por las que el recurso humano se encuentra expuesto para que este sea estudiado y posteriormente controlado, mejorando así los resultados de atención al cliente y la calidad de vida del mismo personal que labora en este tipo de organizaciones, tal como explica Baseman et al. (2018). En este sentido, es posible localizar la problemática que enfrentan los centros de llamado relacionada con el ausentismo del personal, por lo que se hace necesario aportar desde la academia un estudio que permita comprender este fenómeno. Por ello, este artículo de investigación se desarrolla con el objeto de analizar los factores que intervienen en el nivel ausentismo del personal que labora en un centro de llamado de la ciudad de Barranquilla (Colombia).

\section{OTROS ANTECEDENTES}

Para abordar los diferentes antecedentes de la variable objeto de estudio, es importante hablar sobre el ausentismo laboral; este se refiere al fenómeno que tiene un impacto negativo en el normal desarrollo de la empresa, lo que reducirá la productividad de la empresa y aumentará los costos (especialmente el tiempo) porque se debe dedicar más tiempo y personal a otras funciones., según aportan Burgos, Faggioni y Pérez (2018). Por otra parte, Rodríguez et al. (2019) se refiere al ausentismo como la pérdida de tiempo de trabajo es el resultado de que los trabajadores no se presenten en la empresa para realizar sus funciones dentro de la organización.

De acuerdo con Camarota y Pardiñas (2015), se considera que el ausentismo laboral se refiere a una aplicación del término ausentismo aplicado al área laboral, la cual se relaciona hacia el incumplimiento de las jornadas laborales por parte de los trabajadores. Autores como Baptista et al (2016) explican que la Organización Internacional del Trabajo (OIT) lo define como la inasistencia de un trabajador a su sitio de trabajo el cual se tenía estipulado iba a asistir; excluyendo los periodos vacacionales, huelgas o el ausentismo de causa medica que se reconoce como una incapacidad personal que se excepciona para las derivadas de cárcel o embarazo.

Asimismo, su contribución debería tener en cuenta que las tres excepciones mencionadas por la OIT deberían incluirse en la medición del absentismo a fin de establecer normas más claras a nivel internacional. El absentismo laboral es un fenómeno, que es un tema muy importante para el normal desarrollo de la empresa y una de las mayores preocupaciones de los empresarios, porque obviamente es un tema común debido a su existencia. Casi todas las empresas tienen un problema en su negocio, un problema de difícil solución por la complejidad que lo rodea: la causa, las consecuencias y el impacto.

En primer lugar, aportan Souza et al. (2020) que esto representa para las empresas, este es un problema grave porque aumenta los costos y dificulta la planificación y la consecución de sus objetivos. Para los trabajadores expresa una situación de disconformidad de su relación asalariada, a la vez que también puede impactar en sus percepciones económicas. Para el Estado disminuye los ingresos de la Seguridad Social. En este sentido se pueden vislumbrar un poco las consecuencias que trae el ausentismo en las empresas pues, tal como aportan Dula et al (2019) bajan los índices de calidad y elevan los costes para la empresa, así mismo pudiendo representar la pérdida del capital humano. Ciertamente, el ausentismo dentro de las empresas trae una importante cantidad de implicaciones, entre ellas se puede establecer la siguiente clasificación basándose en Arregui (2018) tal como se observa en la tabla 1.

Por tanto, al analizar la dinámica laboral de las organizaciones, varios autores confirmaron la conexión entre diversas causas y variables, que van desde la meta más objetiva (como el costo incurrido) hasta la meta secundaria (como la presión de trabajo). La cultura organizacional con factores humanos y transmitida de generación en generación, la realidad de la organización y otros factores relacionados han generado problemas que no aparecerán de manera aislada en ninguna empresa. Determinar la causa y formular un plan de acción dirigido a minimizar o eliminar la causa raíz, y sus consecuencias, para de este modo orientar su prevención. 
Tabla 1. Causas del ausentismo laboral

\begin{tabular}{|l|l|}
\hline Nivel & Conceptualización \\
\hline Laboral: & $\begin{array}{l}\text { El absentismo se da ante tareas cansadas, monótonas, repetitivas e incompletas, } \\
\text { que reducen enormemente el interés y la motivación de las personas, y llevando a } \\
\text { una situación de alienación, de la que intentó deshacerse. }\end{array}$ \\
\hline Económica: & $\begin{array}{l}\text { Cuando los trabajadores se enfrentan a que la relación salarial no es para nada } \\
\text { satisfactoria, aumenta el absentismo, lo que los motivará más para realizar las } \\
\text { tareas }\end{array}$ \\
\hline Social: & $\begin{array}{l}\text { Cuando los trabajadores enfrentan el desempleo o la crisis económica, la tasa de } \\
\text { absentismo disminuirá, y los esfuerzos para reducir la tasa de absentismo son el } \\
\text { principal instinto de autoprotección. }\end{array}$ \\
\hline
\end{tabular}

En este punto, resulta interesante revisar modelos teóricos como los de Muchinsky (1977). y Marshall, (2019) que consideran al ausentismo como una respuesta de adaptación del trabajador con su ambiente de trabajo, donde el ausentismo se presenta a partir de la pregunta del que hacen los colaboradores ante bajos niveles de satisfacción. Al abordar caso de los centros de llamado, se considera que los bajos niveles de satisfacción de los trabajadores traen graves consecuencias como las renuncias voluntarias, baja productividad y los altos niveles de ausentismo. A su vez, al abordar el caso latinoamericano, estudios como los de Arregui (2018) permiten observar lo mismo, donde se considera que para medir y comprender el ausentismo en los centros de llamado se debe abordar desde la diversidad de causales; resaltando la satisfacción, y otros elementos que definen las dinámicas de la organización y la sociedad misma.

\section{METODOLOGÍA}

El presente artículo de investigación se desarrolla desde el enfoque cuantitativo; siendo este un estudio de tipo correlacional con un diseño de campo no experimental; pues a través de este se busca establecer el nivel de asociación entre el ausentismo y los diversos factores sociodemográficos que pueden influir en este, donde las mediciones fueron realizadas en su lugar de origen y sin dar manipulación de ningún tipo a las variables. Sobre la muestra seleccionada, se destaca que esta se constituyó por 65 sujetos que laboran en un centro de llamada de la ciudad de Barranquilla.

Del mismo modo, con relación con el instrumento utilizado se encuentra basado en el realizado por Arregui (2018) el cual tomó como referencia los constructos de herramientas existentes, validadas y relacionadas a las variables donde el estudio cualitativo arrojó los resultados más significativos; se destaca que el presente estudio realizó modificaciones al instrumento para adaptarlo a las necesidades del contexto. Dicho instrumento contiene dos partes; una primera sección de 6 ítems donde ser coloca la información sociodemográfica de los encuestados (edad, sexo, nivel de estudios, miembros del grupo familiar, tiempo en la empresa y cargo); y una la segunda con 36 preguntas y se distribuye en cuatro dimensiones: Riesgo (sensación de riesgo del trabajador), Cultura organizacional (los elementos culturales que hacen parte de la estructura organizacional), Engagement (apego de los trabajadores con su puesto de trabajo y la empresa) y Ausentismo (capacidad de ausentismo laboral y conductas relacionadas con ello).

Las 36 preguntas del cuestionario son las siguientes: 1) Sé exactamente lo que se espera de mí en el trabajo, 2) Las políticas generales dadas por la gerencia facilitan el buen desempeño, 3) Mi superior/a directo hace cosas para motivarnos 4) ¿Con qué frecuencia tiene conflictos con su supervisor/a?, 5) Mi supervisor se preocupa de mi bienestar personal, 6) Mi equipo disfruta de estatus o prestigio dentro de la organización, 7) La relación entre compañeros de trabajo es buena, 8) ¿Ha sufrido dolores de cabeza durante los últimos seis meses? , 9) ¿Le ocurre que no tiene tiempo suficiente para completar su trabajo? ¿Con qué frecuencia tiene conflictos con los clientes?, 10) Presto mucha atención a los clientes, pero obtengo poco agradecimiento a cambio, 11) ¿El ritmo de trabajo es alto durante toda la jornada?, 12) ¿Las exigencias de trabajo mental en su cargo son altas?, 13) ¿Los métodos de control y evaluación de mi gestión se fijan en base a metas realistas?, 14) ¿Conozco los criterios con los que me evalúan?, 15) ¿Ha tenido algún problema de salud a causa de su trabajo? , 16) ¿Puede abandonar su trabajo por unos minutos sin necesidad de que le sustituyan?, 17) La mayoría de los miembros de este grupo están muy comprometidos con su trabajo, 18) Existe un conjunto de valores claro y consistente que rige la forma en que nos conducimos, 19) Existe en el área un estilo de liderazgo característico con un conjunto de prácticas distintivas, 20) Los comentarios y recomendaciones de nuestros clientes conducen a menudo a introducir cambios, 21) En mi trabajo me siento Ileno/a de energía, 22) Mi trabajo es estimulante e inspirador, 23) Estoy orgulloso/a del trabajo que hago, 24) Me excuso para no ir a trabajar (p.ej: con una lumbalgia) cuando estoy muy cansado, 25) Digo que me encuentro enfermo, aunque me encuentro bien, porque no deseo ir a trabajar, 26) Digo que me encuentro enfermo para no ir a trabajar, pero no voy por razones personales y privadas, 27( Alargo unos minutos el tiempo estipulado que tengo para comer, ir al baño, etc., 28) Hago una pausa en mi trabajo con la excusa de 
ir al médico, a RRHH, etc. 30) ¿Cuántos días en los últimos doce meses ha faltado al trabajo?, 31) notifico de manera apropiada cuando me debo ausentar en al trabajo, 32) me preocupa cuando debo ausentarme en el trabajo, 33) existe un proceso de control cuando me ausento constantemente, 34) busco motivos para ausentarme en el trabajo, 35) repongo las horas o los procesos que deje de entender mientras estuve ausente y 36) No me siento obligado ni responsable por cumplir con mis labores en el trabajo. A su vez, el cuestionario cuenta con una escala de valoración de tipo Likert que se desarrolla de la siguiente manera en la tabla 2:

Tabla 2. Escala del instrumento

\begin{tabular}{|c|c|c|}
\hline Valor \# & Frecuencia de la respuesta & Valoración del baremo \\
\hline 1 & Nunca & Deficiente \\
\hline 2 & Casi nunca & Malo \\
\hline 3 & A veces & Regular \\
\hline 4 & Casi siempre & Bueno \\
\hline 5 & Siempre & Muy bueno \\
\hline
\end{tabular}

Es de destacar que, con respecto a la dimensión de ausentismo del instrumento, dichos resultados son colocados a la inversa de la escala. Se menciona a su vez, que para validar y determinar confiabilidad se hizo necesario realizar cambios al instrumento, especialmente enfocándose en crear sistema de baremo más eficiente que el utilizado por Arregui (2018). Realizados los cambios, se validó el instrumento por 5 expertos en el área de los recursos humanos y se determinó su confiabilidad por medio del Alfa de Cronbach, donde se obtuvo un puntaje considerado como bueno según Taber (2018) en su estudio sobre la recolección de información en estudios desde los instrumentos investigativos; siendo este de 0,83. Por último, con relación al análisis de los datos, se destaca que estos se hicieron empleando la estadística descriptiva por medio de las frecuencias, los porcentajes y el coeficiente de Correlación de Pearson.

\section{RESULTADOS}

En primera instancia, para desarrollar el proceso de análisis de los resultados, se realizó una caracterización de los principales factores sociodemográficos de los sujetos objeto de estudio. A partir de los resultados obtenidos en dicho proceso, se procedió a elaborar un perfil con los elementos más representativos de la población en la figura 1. Posterior a la elaboración del perfil característico de los sujetos, se procede al análisis de las dimensiones del instrumento aplicado para reconocer los principales factores que intervienen en el ausentismo personal que labora en el centro de llamado objeto de estudio son los siguientes, tal como se observan en la figura 2:

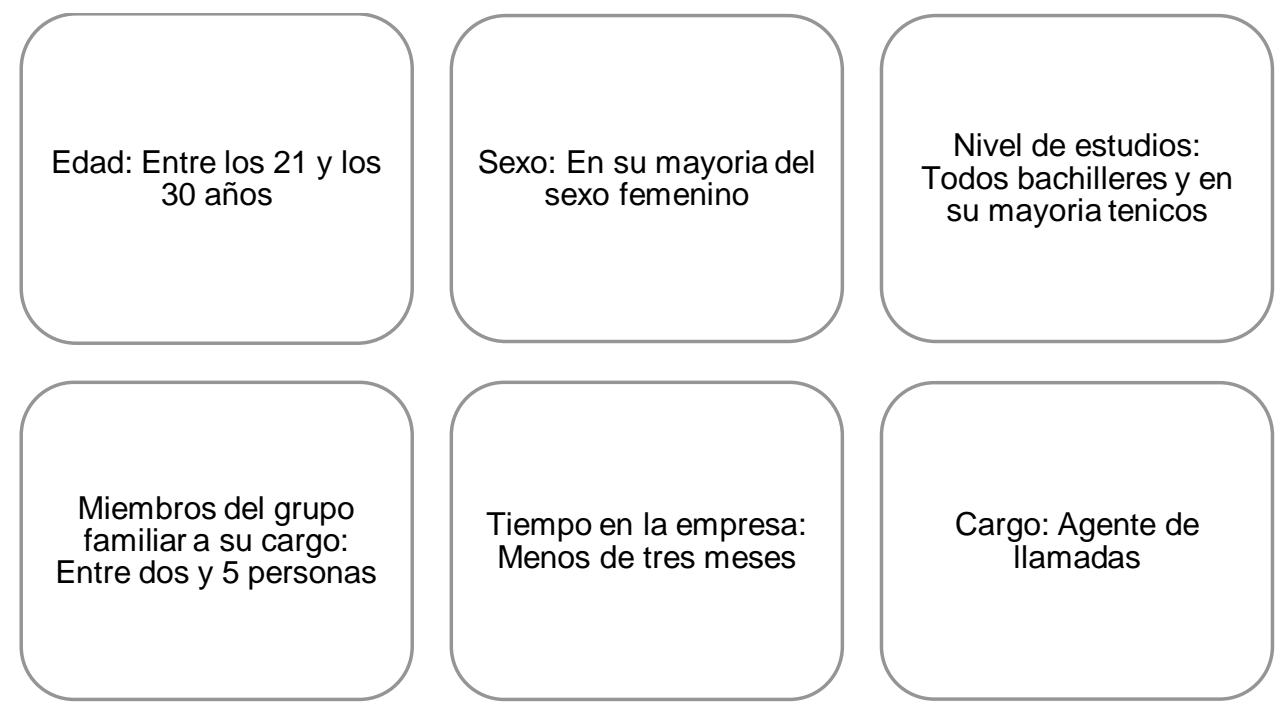

Fig. 1. Perfil sociodemográfico de los sujetos 


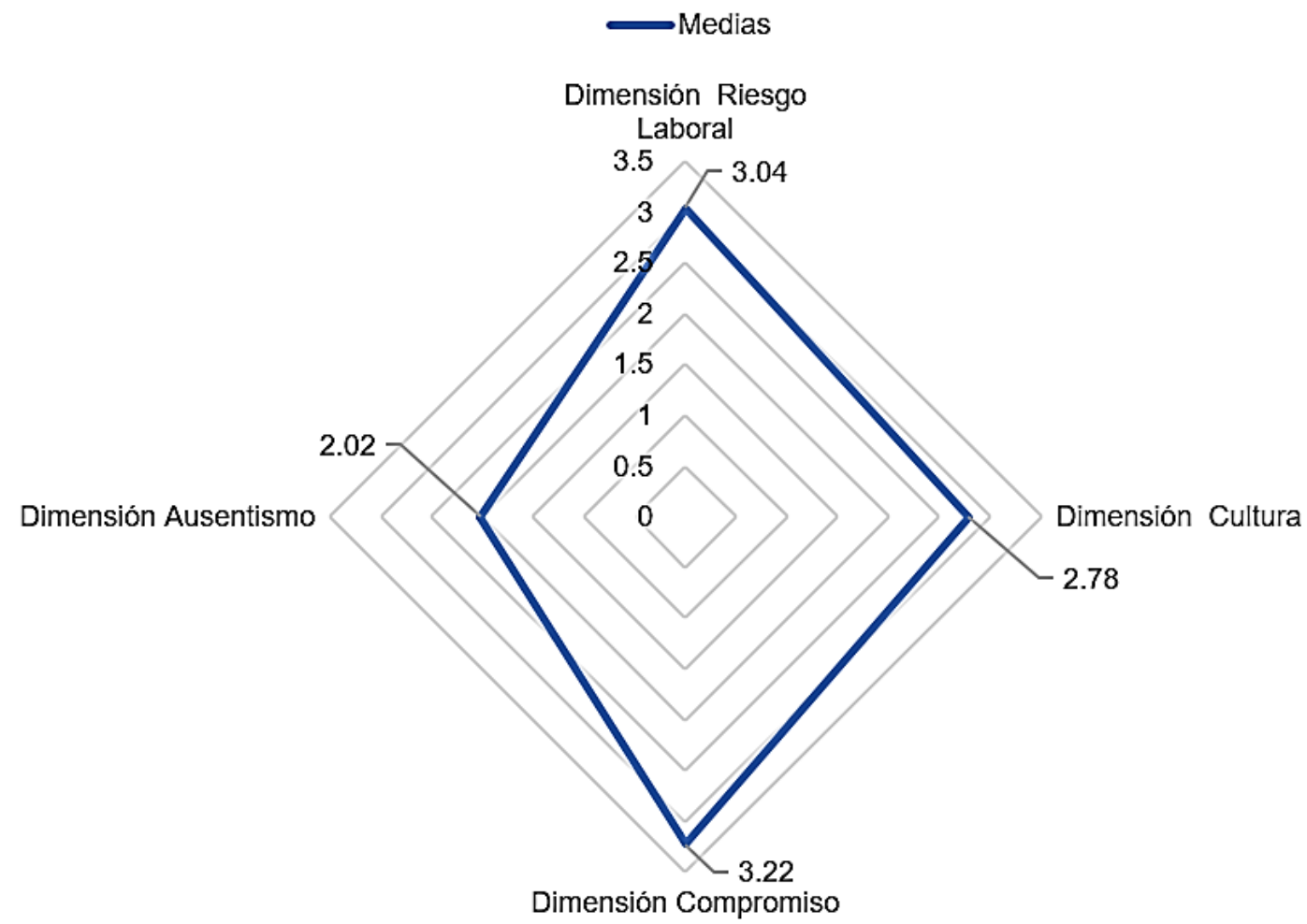

Fig. 2. Resultados de las dimensiones por medias

Tomando en cuenta la figura anterior, se puede observar como las medias registradas en cada uno de los indicadores se encontró en un rango de 2,02 a 3,22 dentro del puntaje del instrumento; tomando en cuenta que los valores más bajos describen niveles negativos de cada uno de los indicadores. En vista de ello, se puede apreciar como la dimensión de engagement conto con la media más alta de 3,22 por lo que se puede considerar que los niveles de esta son positivos; seguido de la dimensión de riesgo laboral la cual tuvo una media de 3,04, siendo esta también positiva, pero con niveles bajos dentro de este mismo rango. Del mismo modo, la dimensión de cultura obtuvo una media de 2,78 la cual ya se encuentra dentro de un rango negativo, se seguido de la dimensión ausentismo 2,02 la cual también conto con una media cuyos valores responden a niveles negativos de esta dimensión. Esto se observa del mismo en siguiente tabla 3:

Tabla 3. Media por dimensión y media total

\begin{tabular}{|l|l|}
\hline Dimensión & Media \\
\hline Dimensión Riesgo Laboral & 3,04 \\
\hline Dimensión Cultura & 2,78 \\
\hline Dimensión Engagement & 3,22 \\
\hline Dimensión Ausentismo & 2,02 \\
\hline Media total & 2,76 \\
\hline
\end{tabular}

Al observar el cuadro anterior se puede determinar que la media total obtenida en el instrumento aplicado fue de 2,76 la cual es un valor que representa niveles negativos sobre las dimensiones del ausentismo descritas en el instrumento aplicado, pudiendo considerar así que la organización donde ser realizo la medición se encuentra en una situación desfavorable con respecto a lo mencionado. Una vez realizados el perfil sociodemográfico y el análisis de las medias del instrumento se procede a comparar dichos resultados para lograr verificar si existe o no algún tipo de asociación entre estos elementos. De este modo, se requirió calcular la relación existente entre las variables de estudios; para ello se aplicó el Coeficiente de Correlación de Pearson.

Se destaca que dentro de la variable sociodemográfica del cargo que fue tomada en este estudio para el análisis de correlación, se hizo por medio de una valoración inversa, a su vez la variable de sexo no fue considerada pues casi la totalidad de las personas encuestadas son mujeres. Es de considerar que para que una correlación se considere como significativa, es necesario que este nivel de asociación sea al 0,01, donde si este número es positivo el nivel de asociación tendrá este comportamiento y si es negativo así también será la relación entre las variables, estos se representaran en una tabla con el signo de "*"; tal como se aprecia en la tabla 4. 
Tabla 4. Correlaciones; ${ }^{* * \star}$ La correlación es significativa al nivel 0,01 (bilateral).

\begin{tabular}{|c|c|c|c|c|c|c|c|}
\hline & & Aus. & Edad & \begin{tabular}{|l|} 
Nivel de \\
estudios
\end{tabular} & \begin{tabular}{|l|} 
Miembros \\
grupo familiar \\
a su cargo \\
\end{tabular} & $\begin{array}{l}\text { Tiempo en el } \\
\text { área de trabajo }\end{array}$ & Cargo \\
\hline \multirow[t]{3}{*}{ Aus } & Correlación de Pearson & 1 & $0.401^{* *}$ & -0.089 & 0.031 & $0.967^{\star \star \star}$ & $0.628^{* * *}$ \\
\hline & Sig. (bilateral) & & 0.001 & 0.487 & 0.810 & $<.001$ & $<.001$ \\
\hline & $N$ & 65 & 65 & 65 & 65 & 65 & 65 \\
\hline \multirow[t]{3}{*}{ Edad } & Correlación de Pearson & $0.401^{* *}$ & 1 & -0.072 & -0.040 & $0.348^{* *}$ & $0.331^{* *}$ \\
\hline & Sig. (bilateral) & 0.001 & & 0.576 & 0.760 & 0.005 & 0.008 \\
\hline & $N$ & 65 & 65 & 65 & 65 & 65 & 65 \\
\hline \multirow{3}{*}{$\begin{array}{l}\text { Nivel de } \\
\text { estudios }\end{array}$} & Correlación de Pearson & -0.089 & -0.072 & 1 & 0.038 & -0.101 & -0.076 \\
\hline & Sig. (bilateral) & 0.487 & 0.576 & & 0.767 & 0.431 & -0.076 \\
\hline & $\mathrm{N}$ & 65 & 65 & 65 & 65 & 65 & 65 \\
\hline \multirow{3}{*}{$\begin{array}{l}\text { Miembros } \\
\text { grupo familiar }\end{array}$} & Correlación de Pearson & 0.031 & -0.040 & 0.038 & 1 & 0.006 & 0.002 \\
\hline & Sig. (bilateral) & 0.810 & 0.760 & 0.767 & & 0.963 & 0.989 \\
\hline & $\mathrm{N}$ & 65 & 65 & 65 & 65 & 65 & 65 \\
\hline \multirow{3}{*}{$\begin{array}{l}\text { Tiempo en el } \\
\text { área de } \\
\text { trabajo }\end{array}$} & Correlación de Pearson & $0.967^{* * *}$ & $0.348^{* *}$ & -0.101 & 0.006 & 1 & $0.604^{* * *}$ \\
\hline & Sig. (bilateral) & $<.001$ & 0.005 & 0.431 & 0.963 & & $<.001$ \\
\hline & $N$ & 65 & 65 & 65 & 65 & 65 & 65 \\
\hline \multirow[t]{3}{*}{ Cargo } & Correlación de Pearson & $0.628^{* \star *}$ & $0.331^{* *}$ & -0.076 & 0.002 & $0.604^{* * *}$ & 1 \\
\hline & Sig. (bilateral) & $<.001$ & 0.008 & -0.076 & 0.989 & $<.001$ & \\
\hline & $N$ & 65 & 65 & 65 & 65 & 65 & 65 \\
\hline
\end{tabular}

Tomando en cuenta lo mostrado en la tabla anterior, se logra observar cómo existe un nivel de asociación significativo entre el ausentismo y las variables sociodemográficas de edad, cargo y tiempo dentro de la organización; esto permite identificar que por mayor sea el cargo, el tiempo dentro de la organización y la edad existe una mayor tendencia de los trabajadores por contar con menor posibilidad de inclinarse hacia el ausentismo. De esta manera, dentro de la organización existe un muy alto porcentaje de trabajadores jóvenes, con poco tiempo dentro de la empresa y que cumplen con el cargo más bajo dentro de la organización; por lo que es preocupante que tres características del perfil de los trabajadores sean elementos predictores de ausentarse de las responsabilidades laborales. Los resultados obtenidos en el presente estudio permiten no solo caracterizar socio demográficamente el perfil de los trabajadores que laboran en el centro de llamado objeto de estudio y evaluar el nivel que dicha organización obtuvo en el cuestionario aplicado, si no que permite establecer los niveles de asociación entre los factores evaluados y los puntajes obtenidos.

De este modo se logró establecer como factores de la edad, tiempo en la empresa y el cargo está altamente relacionados con los altos niveles de rotación y ausentismo que se dan en la empresa. A partir de aquí, se puede establecer una comparación entre los resultados obtenidos por parte de Aregui (2018) quien encontró que los riesgos laborales se presentaron como los elementos que poseen más impacto sobre los niveles de ausentismo, seguido por los elementos de cultura, engagement y clima organizacional. Con base a ello, se debe destacar que en ese estudio no se tomó en cuenta el elemento del clima como una dimensión en sí y se añadió la dimensión de ausentismo. Otro estudio significativo es el de Burgos, Faggioni y Pérez (2018) quienes encontraron que en la mayoría de las empresas con altos índices de rotación simplemente no existen políticas que busque regularlas o disminuirlas, así mismo determinaron que el ambiente laboral figuro como uno de los elementos más representativos.

Ciertamente lo aportado por estos autores cobra realidad cuando se verifica que dentro de la organización objetivo de estudio no solamente no existen políticas claras para disminuir o regular la rotación y el ausentismo dentro de ella, si no que carecen de un sistema claro para llevar controles de dichos indicadores. Estos autores, por la necesidad encontrada consideraron fundamental la elaboración de una propuesta planteándose como meta fundamental un aumento significativo de la productividad organizacional, del bienestar de los empleados dentro de un entorno óptimo, con niveles de estabilidad y con capacidad de reducir los niveles de absentismo del recurso humano. 
A su vez, resulta importante citar a Marshall (2019), quien encontró desde los estilos de liderazgo y la motivación creada hacia los colaboradores la clave para comprender los altos niveles de ausentismo dentro de los centros de llamado. Realmente resulta interesante contrastar con estos estudios que se enfocan hacia los elementos característicos de la organización, pero no profundizan mucho en las características de cada individuo y como estas afectan su relación con la empresa, pues tal como explican diversos autores; la satisfacción del trabajador es un resultado subjetivo de la interacción de cada individuo con la organización. Por último, se puede realizar un contraste con Duarte (2020) quien aplicó un estudio sobre el ausentismo en un centro de llamado de la ciudad de Barranquilla; quien también encontró que los niveles de riesgo laboral dentro de este tipo de organizaciones son sumamente elevados y que el factor de la edad puede ser considerado como un elemento predictor del ausentismo laboral.

\section{DISCUSION FINAL}

En primera instancia, se debe destacar que los altos niveles de ausentismo es una grave problemática a la que se enfrentan hoy en día en muchas empresas, especialmente las que laboran en sector de los centros de llamado por los elevados niveles de tensión que son manejados dentro de estas empresas. De este modo es esencial que las organizaciones reconozcan como esto no solo afectan negativamente a los colaboradores al no percibir sus salarios completos si no que afectan negativamente la estabilidad y calidad de las empresas. Por ello, es preocupante que durante este estudio en sus primeras etapas se buscó indagar los historiales de la organización sobre el tema, a lo cual se encontró que no había bases de datos organizadas.

Una de las barreras más grandes que se lograron identificar en el trascurso del estudio se relaciona con la dificultad de establecer contacto con una empresa del sector que estuviese dispuesta compartir su información, esto ciertamente, al haber analizado los resultados y revisado el comportamiento histórico según la teoría puede deberse a que el sector de los centros de llamado busca no divulgar información negativa para evitar crear mala reputación hacia la posible captación de nuevo personal. Al revisar cada uno de los indicadores del cuestionario, se logra identificar como las dimensiones de cultura y ausentismo contaron con puntajes sumamente bajos; para así reconocer debilidades en estas dos dimensiones dentro de la misma organización. Con relación a las otras dos dimensiones de estabilidad y engagement, se logró comprobar que, aunque estos contaron con niveles positivos dentro de la escala, los niveles obtenidos aun fueron bajos para considerarse excelentes dentro de la empresa.

\section{CONCLUSIONES}

De acuerdo con el trabajo presentado y a los resultados obtenidos, se pueden plantear las siguientes conclusiones principales:

1. Se concluye que el ausentismo presente en el centro de llamada objeto de estudio arrojó resultados elevados, lo cual se puede justificar al momento de analizar los niveles de Riesgo, Cultura organizacional, y Engagement; los cuales mostraron ser a su vez bastante negativos. Esto ciertamente demuestra que este tipo de organizaciones necesitan realizar una revisión sobre las prácticas tomadas sobre los trabajadores para incentivar una cultura organizacional positiva, los niveles de engagement y que los colaboradores no sientan que el riesgo de perder su trabajo es alto.

2. Del mismo modo, se puede concluir que ciertamente los centros de llamado generan un importante aporte hacia las economías de los países latinoamericanos, permitiendo ampliar las capacidades de empleabilidad en dichas naciones, pero a su vez se considera que es fundamental mejorar las condiciones que estas organizaciones les brindan a los trabajadores, no solo para disminuir los niveles de ausentismo que ciertamente genera un efecto negativo en la rentabilidad de la organización y la prestación de sus servicios, sino que también tiene grandes consecuencias sobre la calidad de vida de los colaboradores.

3. Se concluye a su vez que una de las limitaciones más grandes con las que contó el presente estudio es el acceso la información del centro de llamada, pues este tipo de instituciones son bastante reservadas con esta información, siendo considerada en muchos otros centros de llamado de la ciudad de Barranquilla como confidencial.

4. Se recomienda que se sigan desarrollando diversas investigaciones sobre el ausentismo de los trabajadores en los centros de llamado, partiendo de diversos elementos organizacionales que se relacionen con la satisfacción y la calidad de vida; de modo que se puedan visionar diversas propuestas de mejora para las necesidades de este sector en específico. 


\section{REFERENCIAS}

Agnihotri, R., Dingus, R., Hu, M. y Krush, M., Social media: Influencing customer satisfaction in B2B sales. https://doi.org/10.1016/j.indmarman.2015.09.003, Industrial Marketing Management, 53(1) (2016)

Arregui, A., El ausentismo laboral. Caso: “Área Call Center institución financiera privada del Ecuador”. Universidad Andina Simón Bolívar (2018).

Duarte, J., Análisis de los niveles de ausentismo laboral en un call center de venta de productos y servicios de la ciudad de Barranquilla. Corporación Universitaria Latinoamericana (2020).

Baptista, D., Méndez, S., Zunino, N. y Baptista, D., Ausentismo Laboral, Universidad de la República, 6(8) (2016).

Burgos, L., Faggioni, A. y Pérez, J., Gestión de talento humano en relación al ausentismo y rotación de personal en las empresas constructoras de la ciudad de Guayaquil, Observatorio de la Economía Latinoamericana, (2018).

Camarota, R. y Pardiñas, V., El ausentismo laboral de causa médica en la policía nacional uruguaya, Biomedicina, 10(1), 34-53 (2015).

Cheshin, A., Amit, A. y Van Kleef, G. A., The interpersonal effects of emotion intensity in customer service: Perceived appropriateness and authenticity of attendants' emotional displays shape customer trust and satisfaction. https://doi.org/10.1016/j.obhdp.2017.10.002, Organizational Behavior and Human Decision Processes, 144 (2018).

Deery, S., Walsh, J., Zatzick, C. y Hayes, A., Exploring the relationship between compressed work hours satisfaction and absenteeism in front-line service work. https://doi.org/10.1080/1359432X.2016.1197907, European Journal of Work and Organizational Psychology, 26(1), $42-52$ (2017).

Dula, T., Abara, G. y Reddy, P., The Assessment of Causes and Consequences of Medical Staff Absenteeism and Turnover in Arba Minch General Hospital. 10.7176/JHMN/58-04, Assessment, 58 (2019).

Elmorshidy, A., Mostafa, M., El-Moughrabi, I. y Al-Mezen, H., Factors influencing live customer support chat services: an empirical investigation in Kuwait, Journal of theoretical and applied electronic commerce research, 10(3), 63-76 (2015).

Feldhaus, C., Souza, R. y otros 4 autores, Association between workload and absenteeism in nursing technicians. https://doi.org/10.1590/1980-265x-tce-2018-0307, Texto \& Contexto-Enfermagem, 28 (2019).

Frick, B., Simmons, R. y Stein, F., The cost of shift work: Absenteeism in a large German automobile plant. 10.1177\%2F2397002218788839, German Journal of Human Resource Management, 32(3-4) 236-256 (2018).

García, J., Durán, S., Parra, M. y Márceles, V., Dirección estratégica del talento humano para el fomento de valores en los cuerpos policiales venezolanos, Revista Espacios, 38(32) (2017).

Garcia, M., Sukier, H. y otros 4 autores, Marketing estratégico: una mirada desde el contexto de la empresa familiar, Revista Espacios, 39(44) (2018).

Hernandez-Sánchez, I., Parra, M. y otros 4 autores, Comportamiento Organizacional Ciudadano (COC) como ejemplo de participación generador de un diálogo de saberes, Revista Espacios, 39(7) (2018).

Luengo, C., Moraga, S. y otros 3 autores, Fatiga laboral y ausentismo en el trabajo en mujeres auxiliares de enfermería de un hospital de alta complejidad. https://doi.org/10.1590/1413-81232020251.28832019, Chile, Ciência \& Saúde Coletiva, 25, 243-249 (2019).

Marshall, G., Strategies to Reduce Job Dissatisfaction within 911 Call centers. Walden University (2019).

Meesala, A. y Paul, J., Service quality, consumer satisfaction and loyalty in hospitals: Thinking for the future. http://dx.doi.org/10.1016/j.jretconser.2016.10.011, Journal of Retailing and Consumer Services, 40, $261-269$ (2018).

Muchinsky, P., Employee absenteeism: A review of the literature, Journal of Vocational Behavior, 10(3), 316-340 (1977).

Parra, M., Duran, S. y otros 8 autores, Factores del clima organizacional existentes en el área administrativa en empresas de Barranquilla, Revista Espacios, 39(50) (2018).

Payares, K., Parra, M., Navarro, E. y Naranjo, O., Mercadeo interno en las pequeñas y medianas empresas del sector salud de Barranquilla (Colombia). http://dx.doi.org/10.4067/S0718-07642020000100123, Información tecnológica, 31(1), 123-132 (2020).

Pillay, K., Buitendach, J. y Kanengoni,, H., Psychological capital, job demands and organisational commitment of employees in a call centre in Durban, South 117 Africa. 10.4102/sajhrm.v12i1.599, Human Resource Management Journal, 12(1) (2014).

Pinho, H., Santos, J. y otros 3 autores, Transtornos de ansiedade como causa de afastamentos laboráis. https://doi.org/10.1590/2317-6369000021417, Revista Brasileira de Saúde Ocupacional, 44, 1-8 (2019).

Rodríguez, L., Artazcoz, L. y otros 3 autores, Joint effect of paid working hours and multiple job holding on work absence due to health problems among basic education teachers in Brazil: the Educatel Study. https://doi.org/10.1590/0102311X00081118, Cadernos de saude publica, 35 (2019).

Rompu, J., Wahyu, A. y Abadi, M. Y., The Effect of Occupational Stress, Quality of Worklife and Organizational Climate on Officials' Work Satisfaction of Regional Public Hospital of Undata Palu. http://dx.doi.org/10.5958/09765506.2018.00228.0, Indian Journal of Public Health Research \& Development, 9(3) (2018). 
Souza, A., Fernandes, R., Carvalho, F. y Porto, L., Fatores associados ao presenteísmo em trabalhadores da industria. https://doi.org/10.1590/2317-6369000003118, Revista Brasileira de Saúde Ocupacional, 45 (2020).

Stabio, J., Factores que impulsan el ausentismo en el centro de contacto. El caso de la empresa atento de la ciudad de Córdoba, Argentina. Universidad Católica de Córdoba (2014).

Taber, K. S., The use of Cronbach's alpha when developing and reporting research instruments in science education. https://doi.org/10.1007/s11165-016-9602-2, Research in Science Education, 48(6) (2018).

Zablah, A. R., y otros 3 autores, A cross-lagged test of the association between customer satisfaction and employee job satisfaction in a relational context, Journal of Applied Psychology, 101(5) (2016). 